\title{
STELLAR AND MOLECULAR RADII OF A MIRA STAR: FIRST OBSERVATIONS WITH THE KECK INTERFEROMETER GRISM
}

\author{
J. A. Eisner, ${ }^{1,2}$ J. R. Graham, ${ }^{1}$ R. L. Akeson, ${ }^{3}$ E. R. Ligon, ${ }^{4}$ M. M. Colavita, ${ }^{4}$ G. Basri, ${ }^{1}$ \\ K. Summers, ${ }^{5}$ S. Ragland, ${ }^{5}$ AND A. Booth ${ }^{4}$ \\ Received 2006 October 9; accepted 2006 November 8; published 2006 December 7
}

\begin{abstract}
Using a new grism at the Keck Interferometer, we obtained spectrally dispersed $(R \sim 230)$ interferometric measurements of the Mira star R Vir. These data show that the measured radius of the emission varies substantially from 2.0 to $2.4 \mu \mathrm{m}$. Simple models can reproduce these wavelength-dependent variations using extended molecular layers, which absorb stellar radiation and reemit it at longer wavelengths. Because we observe spectral regions with and without substantial molecular opacity, we determine the stellar photospheric radius, uncontaminated by molecular emission. We infer that most of the molecular opacity arises at approximately twice the radius of the stellar photosphere.
\end{abstract}

Subject headings: circumstellar matter — instrumentation: interferometers — stars: AGB and post-AGB stars: individual (R Virginis) — techniques: interferometric — techniques: spectroscopic

\section{INTRODUCTION}

Mira variables are asymptotic giant branch (AGB) stars that show long-period (hundreds of days) photometric variability (e.g., Feast et al. 1989). These stars typically lose mass at a rapid rate $\left(\sim 10^{-6} M_{\odot} \mathrm{yr}^{-1}\right)$, and this material condenses into heavy elements, molecules, and dust (e.g., Willson 2000). Since AGB stars account for most of the mass loss in the Galaxy, their circumstellar environments are the likely factories in which many constituents of planets, and life, are produced.

Separating molecular emission and absorption from stellar photospheric emission is important for constraining properties of molecular layers and the underlying stars. The opacity and location of gaseous layers constrain the temperature structure in the circumstellar environments of Mira stars; this directly affects the dust condensation radius and in turn the mass-loss rate. Measurements of stellar radii and temperatures uncontaminated by overlying shells of molecular emission or absorption are needed to estimate the location of Mira stars in the H-R diagram. These quantities also constrain period-mass-radius relations, with direct implications for pulsational properties (e.g., Bowen 1988).

Historically, the circumstellar environments of Mira stars have been studied through high-resolution infrared spectroscopy, which probed layers of $\mathrm{CO}$ and $\mathrm{H}_{2} \mathrm{O}$ above the stellar photospheres (e.g., Hinkle et al. 1982). Interferometric imaging, combined with spectroscopy, has more recently allowed spatially resolved measurements of radio-wavelength continuum and spectral line emission (e.g., Reid \& Menten 1997; Diamond \& Kemball 2003), mid-IR dust and molecular emission (e.g., Weiner et al. 2003), and optical/near-IR photospheric and molecular emission (e.g., Ireland et al. 2004b; Thompson et al. 2002). These observations showed that molecular emission lies

\footnotetext{
Department of Astronomy, University of California at Berkeley, Berkeley, CA; jae@astro.berkeley.edu.

${ }^{2}$ Miller Fellow.

${ }^{3}$ Michelson Science Center, California Institute of Technology, Pasadena, CA. CA.

${ }^{4}$ Jet Propulsion Laboratory, California Institute of Technology, Pasadena,

${ }^{5}$ W. M. Keck Observatory, California Association for Research in Astronomy, Kamuela, HI.
}

above the stellar photosphere and that different molecules lie at different stellocentric radii.

Previous near-IR interferometric observations, which probed the hottest $(\gtrsim 1000 \mathrm{~K}$ ) circumstellar emission, used either very low spectral resolution $(R \lesssim 25$; e.g., Thompson et al. 2002 ; Mennesson et al. 2002) or a few narrowband filters designed to probe certain molecular lines or nearly line-free regions of the spectrum (e.g., Perrin et al. 2004). These observations provided evidence for different spatial distributions of stellar continuum and molecular radiation but lacked the spectral resolution to clearly separate various components. With higher spectral resolution $(R \gtrsim 100)$, one can distinguish contributions from $\mathrm{H}_{2} \mathrm{O}, \mathrm{CO}$, and the stellar photosphere, and can even discern several of the $\mathrm{CO}$ band heads. Higher spectral resolution data therefore allow an estimate of the relative spatial distributions of molecular emission and stellar continuum emission in Mira stars.

Here we present the first observations with a new grism at the Keck Interferometer (KI), which enables an order of magnitude higher spectral resolution $(R \sim 230$ across the $K$ band $)$ than previous studies. We observed the short-period ( $\sim 145$ days) Mira star R Vir (also known as HD 10994, HR 4808, or IRC 10256). This object varies by $\sim 6 \mathrm{mag}$ in the visible (Kholopov et al. 1985) and $\sim 0.6$ mag in the $K$ band (Whitelock et al. 2000). The distance to R Vir is $\sim 400-500$ pc (Jura \& Kleinmann 1992; Knapp et al. 2003), and the mass-loss rate is estimated to be $\sim 10^{-7} M_{\odot} \mathrm{yr}^{-1}$ (Jura 1994).

\section{EXPERIMENTAL SETUP}

We observed R Vir on UT 2006 May 15 using KI. KI is a fringe-tracking long-baseline Michelson interferometer combining light from the two $10 \mathrm{~m}$ Keck apertures (Colavita \& Wizinowich 2003; Colavita et al. 2003). We measured $K$-band fringes that were spectrally dispersed through a replica grism. The grism has an undeviated wavelength of $2.3 \mu \mathrm{m}$ in the first order, an apex angle and blaze angle of $6.5^{\circ}, 21.36$ grooves $\mathrm{mm}^{-1}$, and an index of refraction of 1.435 (Infrasil). The KI detector has a pixel size of $18.5 \mu \mathrm{m}$ and a camera with a focal length of $\sim 82.5 \mathrm{~mm}$. Thus, the grism provides a spectral resolution of $R=230$ with $4210 \mathrm{~nm}$-wide channels across the $K$ band.

We measured squared visibilities $\left(V^{2}\right)$ for our target and two calibrator stars in each of these spectral channels. The calibrator 
stars are $\mathrm{K} 0-\mathrm{K} 4$ giant stars, with known parallaxes, whose $K$ magnitudes are within 0.5 mag of the target. The system visibility (i.e., the point-source response of the interferometer) was measured with these calibrators, whose angular sizes were estimated by fitting blackbodies to literature photometry. The calibrator data were weighted by the internal scatter and the temporal and angular proximity to the target (Boden et al. 1998). Source and calibrator data were corrected for detection biases as described by Colavita (1999) and averaged into $5 \mathrm{~s}$ blocks. The calibrated $V^{2}$ are the averages of $5 \mathrm{~s}$ blocks in each integration, with uncertainties given by the quadrature addition of the internal scatter and the uncertainty in the system visibility.

We averaged our data to produce a single measurement of $V^{2}$ in each spectral channel. Observations of $\mathrm{R}$ Vir spanned $0.7 \mathrm{hr}$, and the averaging has a negligible effect on the $u-v$ coverage. By comparing our calibrator stars to one another, we see that the channel-to-channel variations are small (Fig. 1). Using HD 107328 (spectral type K0 III) to calibrate HD 111765 (spectral type K4 III), we find a standard deviation of 0.012 in the calibrated $V^{2}$ versus wavelength. Calibrating HD 107328 with respect to HD 111765, we find $\sigma=0.028$. We therefore adopt a channel-to-channel uncertainty of $2 \%$ for our data on R Vir.

The normalization of $V^{2}$ versus wavelength has larger uncertainty than the channel-to-channel uncertainties discussed above. Observations of a binary star with a known orbit show that the calibrated $V^{2}$ has a systematic uncertainty of $\$ 5 \%$. This is a discrepancy in the normalization; if we subtract 0.04 from our measurements, the measured and predicted values of $V^{2}$ are consistent across all channels at approximately the $1 \%$ level. We do not apply this offset to our data but assume that in addition to the $2 \%$ channel-to-channel uncertainties described above, the normalization of $V^{2}$ is uncertain by $\sim 5 \%$.

We used the counts in each channel observed during "foreground integrations" (Colavita 1999) to recover a crude spectrum for our target. We divided the measured flux versus wavelength for $\mathrm{R}$ Vir by the observed flux from an A1 V star (smoothed over the $\mathrm{Br} \gamma$ feature at $2.16 \mu \mathrm{m}$ ) and multiplied by a $9000 \mathrm{~K}$ blackbody to calibrate the spectral bandpass. While our spatially filtered data may contain relatively less flux at shorter wavelengths due to the larger effect of atmospheric turbulence, such slopes should be removed through our spectral calibration. We estimate that the channel-to-channel uncertainties are $\sim 5 \%-10 \%$. However, there may be larger systematic errors due to different $K$ magnitudes of the target star and the A1 V spectral calibrator or changing atmospheric or instrumental conditions between the source and calibrator observations. We normalized our calibrated photometry and then converted to flux units by assuming a $K$ magnitude of 2.0 based on the current visual phase and previously studied $K$-band variability.

\section{MODELING \\ 3.1. Star+Shell}

We begin with a simple model consisting of a star surrounded by a single-temperature shell whose optical depth varies with wavelength (essentially a Schuster-Schwarzschild model). We assume the star emits blackbody emission from a compact continuum-forming atmosphere. We ignore limb darkening, which may affect the stellar radius by 5\% (Welch 1994; Claret et al. 1995); the effect is comparable to the assumed uncertainties in the data.

The free parameters of the model are the stellar angular

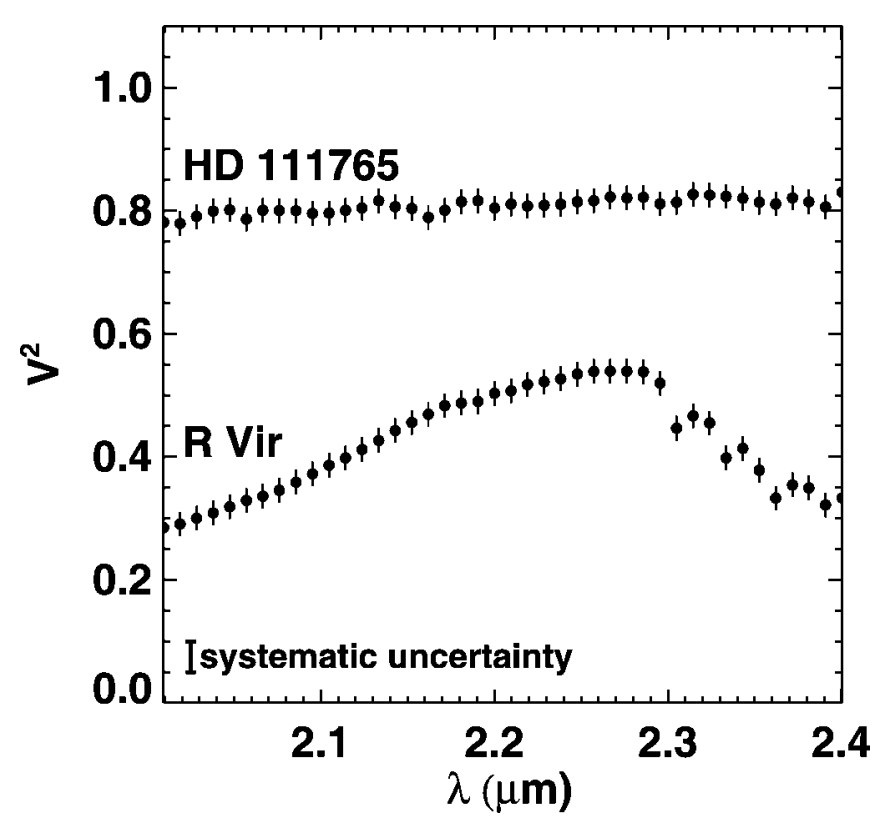

FIG. 1.-Calibrated $V^{2}$ as a function of wavelength for $\mathrm{R}$ Vir and for a $\mathrm{K} 4$ III reference star (note that the reference star is mildly resolved, and thus $V^{2}<1$ ). The plotted error bars correspond to channel-to-channel uncertainties of $2 \%$. We also indicate a $5 \%$ systematic uncertainty in the overall calibration that affects the normalization $(\$ 2)$.

radius and temperature, $\theta_{*}$ and $T_{*}$, the shell radius and temperature, $\theta_{\text {shell }}$ and $T_{\text {shell }}$, and the optical depth of the shell at each wavelength, $\tau_{\lambda}$. Since we must determine the best-fit value of $\tau$ at each observed wavelength, the model has 46 free parameters. We measure $V^{2}$ and flux in each spectral channel, and we have 84 data points with which to constrain these parameters.

This model has been used previously to model narrowband interferometric observations of Mira stars (e.g., Perrin et al. 2004; Weiner 2004) and can be used to estimate the relative spatial distributions of stellar and circumstellar emission. Moreover, comparison of the fitted $\tau_{\lambda}$ with that expected for different molecules (based on laboratory spectroscopy; e.g., Rothman et al. 2005) constrains which molecules are present in the circumstellar environment.

We construct the model by considering sight lines from the center of the star out to the limb of the shell and summing the contributions from each line of sight. The specific intensity of the model includes emission from both the star and the shell for sight lines $\theta \leq \theta_{*}$ :

$$
I_{\nu}(\theta)=B_{\nu}\left(T_{*}\right) e^{-\tau_{\lambda} / \cos \theta}+B_{\nu}\left(T_{\text {shell }}\right)\left(1-e^{-\tau_{\lambda} / \cos \theta}\right) .
$$

For $\theta_{*}<\theta \leq \theta_{\text {shell }}$, only the shell contributes:

$$
I_{\nu}(\theta)=B_{\nu}\left(T_{\text {shell }}\right)\left(1-e^{-2 \tau_{\lambda} / \cos \theta}\right) .
$$

We compute the flux and normalized visibility for a series of annuli of infinitesimal angular width (see, e.g., Eisner et al. 2004) and sum over all annuli to obtain $F_{\nu}$ and $V^{2}$ for the model. We fit our data to the modeled $F_{\nu}$ and $V^{2}$ using a LevenbergMarquardt nonlinear least-squares fitting algorithm.

\subsection{Star + Molecular Layer}

Instead of considering a shell with arbitrary optical depth $\tau_{\lambda}$ (and 42 associated free parameters), we can use molecular 
TABLE 1

Model Parameter Values

\begin{tabular}{cccccc}
\hline \hline Model & $\begin{array}{c}T_{*} \\
(\mathrm{~K})\end{array}$ & $\begin{array}{c}\theta_{*} \\
(\mathrm{mas})\end{array}$ & $\begin{array}{c}T_{\text {shell }} \\
(\mathrm{K})\end{array}$ & $\begin{array}{c}\theta_{\text {shell }} \\
(\mathrm{mas})\end{array}$ & $\begin{array}{c}N_{\text {mol }} \\
\left(\mathrm{g} \mathrm{cm}^{-2}\right)\end{array}$ \\
\hline Star+shell ................ & $3800 \pm 440$ & $1.37 \pm 0.07$ & $1800 \pm 100$ & $2.44 \pm 0.13$ & $\ldots$ \\
Star+molecular layer $\ldots \ldots .$. & $3700 \pm 140$ & $1.40 \pm 0.07$ & $1900 \pm 100$ & $2.42 \pm 0.12$ & $4.3 \pm 0.2$ \\
\hline
\end{tabular}

Note. - Uncertainties listed in the table are the quadrature sums of statistical uncertainties in the fits and assumed $5 \%$ systematic errors.

opacities calculated for stellar atmosphere models (P. Hauschildt 2006, private communication). We replace $\tau_{\lambda}$ with $\tau_{\mathrm{mol}}=N_{\mathrm{col}} \sigma_{\mathrm{mol}}\left(T_{\mathrm{mol}}\right)$, where $\sigma_{\mathrm{mol}}$ is the molecular cross section (as a function of wavelength) for a single layer in the Hauschildt atmospheric model and $N_{\text {col }}$ is the column density of the layer. This column density assumes a mix of different molecules $\left(\mathrm{H}_{2}\right.$, $\mathrm{CO}$, and $\mathrm{H}_{2} \mathrm{O}$, among others), and the relative abundances are determined through self-consistent modeling of a cool stellar atmosphere. Since the relative abundances of $\mathrm{H}_{2}$ and $\mathrm{H}_{2} \mathrm{O}$ and $\mathrm{CO}$ may be lower in a shell than in a dense atmosphere, our fitted column density for the shell may be overestimated. The free parameters for this model are $R_{*}$ and $T_{*}$, the molecular layer radius and temperature, $R_{\mathrm{mol}}$ and $T_{\mathrm{mol}}$, and the column density of the molecules within the layer, $N_{\text {mol }}$.

\section{RESULTS AND CAVEATS}

Our modeling indicates that the stellar radius is $\sim 1.4$ mas $\left(\sim 130 R_{\odot}\right.$, assuming a distance of $\left.450 \mathrm{pc}\right)$ and the stellar temperature is $\sim 3700-3800 \mathrm{~K}$ (Table 1; Fig. 2). The shell of molecular material is cooler and located at a larger radius: $R_{\text {mol }} \sim 2.4$ mas $\left(\sim 230 R_{\odot}\right)$ and $T_{\text {mol }} \sim 1800-1900 \mathrm{~K}$.

We have assumed in our models that the molecular material around R Vir resides in an infinitesimally thin shell. However, material is probably distributed over a range of radii, and different molecules at different temperatures may contribute to the optical depth. Our simple assumption may be partially responsible for differences between the modeled opacity and that expected from molecular line lists (Fig. 3). Deviations from spherical symmetry, not included in our models, may also contribute to differences between models and data.

The inferred temperature of the molecular shell is substantially higher than the expected condensation temperature for dust around Mira stars ( $\sim 500 \mathrm{~K}$; e.g., Rowan-Robinson \& Harris 1982; Hashimoto 1994). Dust at larger radii, but within the 50 mas $(\sim 23 \mathrm{AU})$ field of view of our observations, could contribute some overresolved flux, which would reduce the measured $V^{2}$ and increase the measured flux. This should be a minor effect given the small amount of $K$-band flux from any cool extended dust relative to that in the hot, compact molecular shell modeled above (see, e.g., Eisner et al. 2004).

\section{DISCUSSION}

Our analysis suggests that the molecules (predominantly $\mathrm{H}_{2} \mathrm{O}$ ) responsible for most of the opacity in the circumstellar environment of $\mathrm{R}$ Vir form at approximately twice the radius of the stellar photosphere. The molecular material cannot be supported by hydrostatic forces at this radius. Propagating shocks associated with the pulsation of Mira stars can lead to molecular densities at these radii orders of magnitude larger than predicted by hydrostatic models (e.g., Bowen 1988; Woitke et al. 1999), and shocks thus provide a mechanism for lifting the molecular layer in $\mathrm{R}$ Vir to its observed location.

The radius and temperature of the molecular shell imply radiative equilibrium with the stellar continuum radiation, sug-
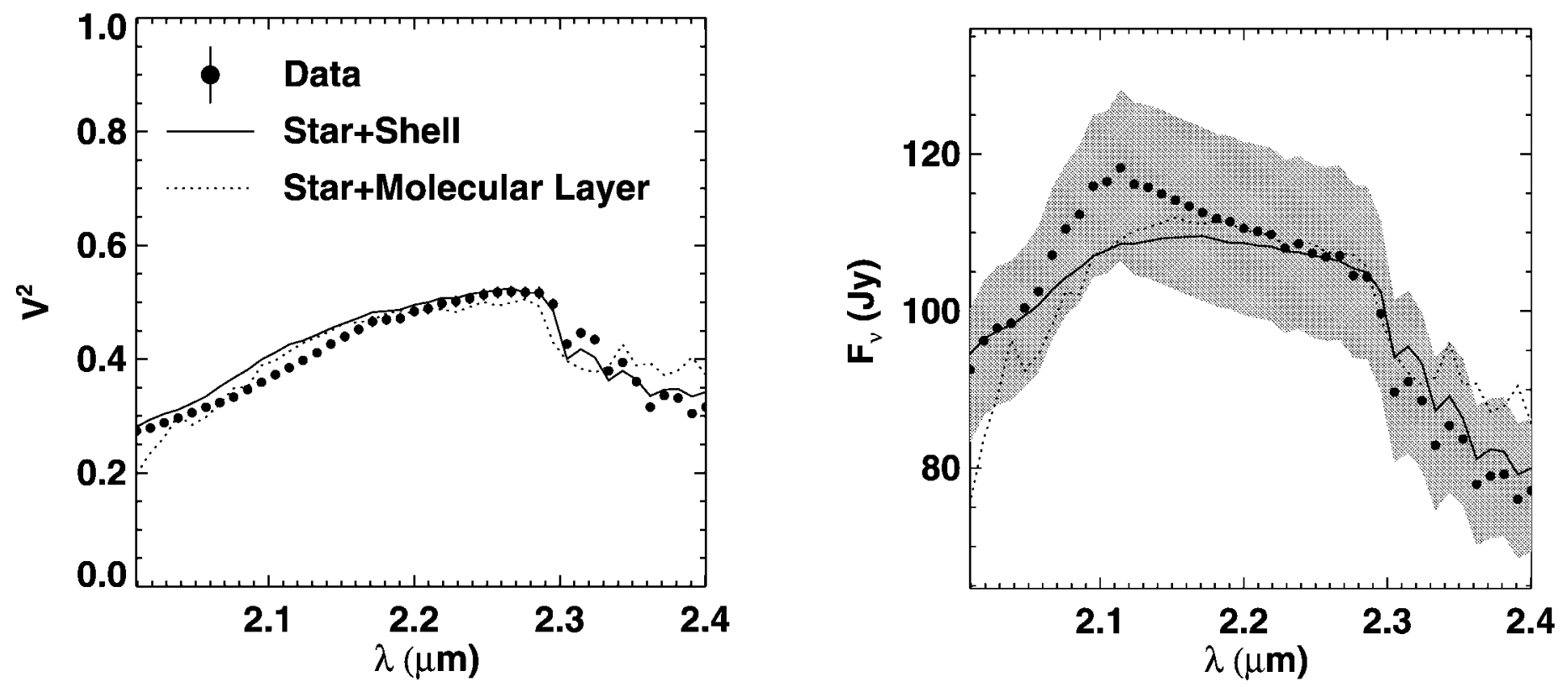

FIG. 2.-Observed $V^{2}$ and fluxes plotted with the predictions of the best-fit models. The shaded region shows assumed $10 \%$ uncertainties for our flux measurements. Solid and dashed lines show the predictions for the star + shell (\$ 3.1) and star+molecular layer (§ 3.2) models, respectively. Water vapor lines blend together at the spectral resolution of these observations and contribute substantial opacity from $\sim 2.0$ to $2.2 \mu \mathrm{m}$ and from $\sim 2.3$ to $2.4 \mu \mathrm{m}$ (e.g., Ludwig 1971). Rovibrational lines of CO contribute opacity between $\sim 2.3$ and $2.4 \mu \mathrm{m}$ (e.g., Rothman et al. 2005), and the four dips in the $V^{2}$ and fluxes between $\sim 2.3$ and $2.4 \mu \mathrm{m}$ occur at the approximate wavelengths of the $\mathrm{CO}$ overtone band heads. 


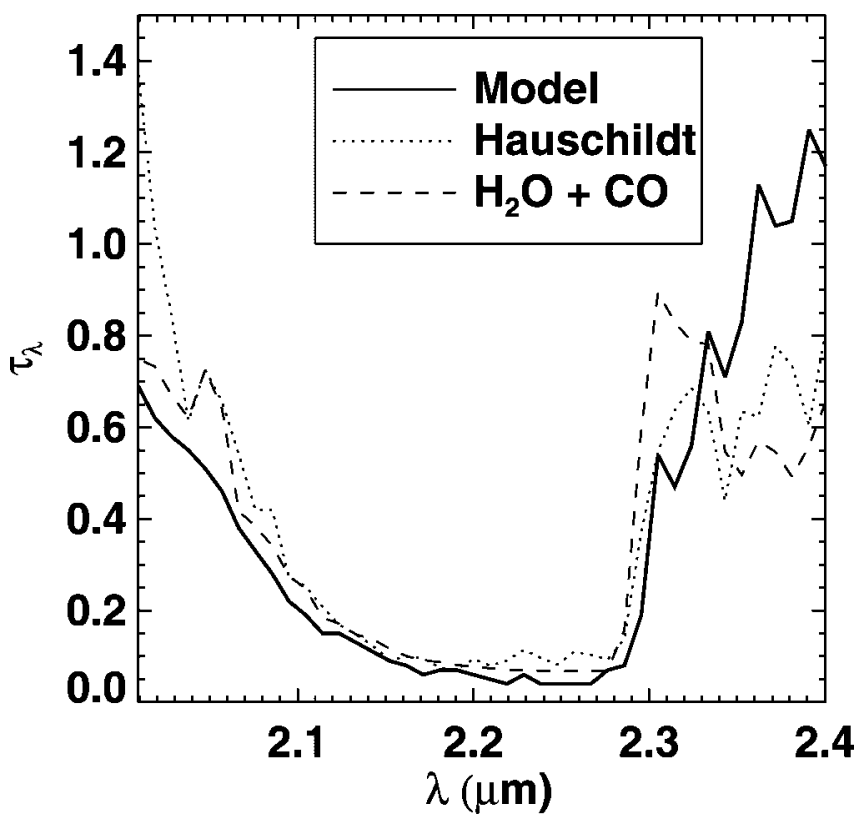

FIG. 3.-Fitted $\tau_{\lambda}$ for the star + shell model (solid line). We also plot $\tau_{\lambda}$, computed for a stellar atmosphere model (P. Hauschildt 2006, private communication) in a layer with $T=1800 \mathrm{~K}$ and $N_{\text {mol }}=5 \mathrm{~g} \mathrm{~cm}^{-2}$, and $\tau_{\lambda}$ based on line lists of $\mathrm{H}_{2} \mathrm{O}$ (Ludwig 1971) and $\mathrm{CO}$ (from HITEMP; Rothman et al. 2005 ) with $T=1800 \mathrm{~K}, N_{\mathrm{H}_{2} \mathrm{O}}=5 \times 10^{20} \mathrm{~cm}^{-2}$, and $N_{\mathrm{CO}}=10^{22} \mathrm{~cm}^{-2}$ (dashed line). These column densities are not fitted, but rather estimated in an order of magnitude sense to produce $\tau_{\lambda}$ close to the modeled value.

gesting that if the shell lies in a postshock region, it has had time to cool. At the molecular densities in such a shell, the radiative cooling time is typically longer than the time between successive shocks. It is more likely that the molecular material in R Vir cooled via expansion; in a rapidly expanding shell associated with a high mass-loss rate, the cooling time is shorter than the time between shocks (e.g., Bowen 1988).

Our model fits imply a stellar radius and temperature of $\sim 130 R_{\odot}$ and $\sim 3800 \mathrm{~K}$. Previous broadband measurements of
Mira diameters (e.g., Haniff et al. 1995), which measured contributions from stellar photospheres and molecular layers, found large stellar radii, which yielded period-mass-radius relations that were consistent with overtone mode pulsation (e.g., Feast 1996). In contrast, the smaller radius determined in this work (and radii determined for other Mira variables from spectrally dispersed or narrowband interferometric observations; e.g., Perrin et al. 2004; Weiner 2004) is consistent with the periodmass-radius relationship expected for fundamental mode pulsations, compatible with other lines of argument that Mira variables should pulsate in the fundamental mode (e.g., Bowen 1988; Wood et al. 1999).

A logical next step is to compare spectrally dispersed interferometry data to dynamic, self-consistent models of Mira variable atmospheres that include the effects of $\mathrm{H}_{2} \mathrm{O}$ opacity. We attempted such comparisons, but currently available models (e.g., Ireland et al. 2004a; Ireland \& Scholz 2006) have longer periods and are either hotter or have larger photometric variability amplitudes than our target. These models predict too much flux and not enough $\mathrm{H}_{2} \mathrm{O}$ to match our data. More appropriate models will become available in the near future (M. Ireland 2006, private communication). Moreover, we will obtain additional observations of $\mathrm{R}$ Vir, enabling a comparison of models and data over a range of pulsation phases

Data presented here were obtained at the W. M. Keck Observatory from telescope time allocated to NASA through the agency's scientific partnership with Caltech and the University of California. The Observatory was made possible by the generous support of the W. M. Keck Foundation. We thank the entire KI team for making these observations possible and acknowledge the role of MSC software in our data acquisition and analysis. We are indebted to P. Wizinowich, G. Vasisht, and L. Hillenbrand for helping to ensure the successful implementation of the grism, and to P. Hauschildt, J. Carr, and M. Ireland for providing models and molecular opacities used in our analysis.

\section{REFERENCES}

Boden, A. F., Colavita, M. M., van Belle, G. T., \& Shao, M. 1998, Proc. SPIE, 3350, 872

Bowen, G. H. 1988, ApJ, 329, 299

Claret, A., Diaz-Cordoves, J., \& Gimenez, A. 1995, A\&AS, 114, 247

Colavita, M. M. 1999, PASP, 111, 111

Colavita, M. M., \& Wizinowich, P. L. 2003, Proc. SPIE, 4838, 79

Colavita, M., et al. 2003, ApJ, 592, L83

Diamond, P. J., \& Kemball, A. J. 2003, ApJ, 599, 1372

Eisner, J. A., Lane, B., Hillenbrand, L., Akeson, R., \& Sargent, A. 2004, ApJ, 613,1049

Feast, M. W. 1996, MNRAS, 278, 11

Feast, M. W., Glass, I. S., Whitelock, P. A., \& Catchpole, R. M. 1989, MNRAS, 241,375

Haniff, C. A., Scholz, M., \& Tuthill, P. G. 1995, MNRAS, 276, 640

Hashimoto, O. 1994, A\&AS, 107, 445

Hinkle, K. H., Hall, D. N. B., \& Ridgway, S. T. 1982, ApJ, 252, 697

Ireland, M. J., \& Scholz, M. 2006, MNRAS, 367, 1585

Ireland, M. J., Scholz, M., \& Wood, P. R. 2004a, MNRAS, 352, 318

Ireland, M. J., Tuthill, P., Bedding, T., Robertson, J., \& Jacob, A. 2004b, MNRAS, 350, 365

Jura, M. 1994, ApJ, 422, 102
Jura, M., \& Kleinmann, S. G. 1992, ApJS, 79, 105

Kholopov, P. N., Samus, N. N., Kazarovets, E. V., \& Perova, N. B. 1985, Inf. Bull. Variable Stars, 2681, 1

Knapp, G. R., Pourbaix, D., Platais, I., \& Jorissen, A. 2003, A\&A, 403, 993 Ludwig, C. B. 1971, Appl. Opt., 10, 1057

Mennesson, B., et al. 2002, ApJ, 579, 446

Perrin, G., et al. 2004, A\&A, 426, 279

Reid, M. J., \& Menten, K. M. 1997, ApJ, 476, 327

Rothman, L. S., et al. 2005, J. Quant. Spectrosc. Radiat. Transfer, 96, 139

Rowan-Robinson, M., \& Harris, S. 1982, MNRAS, 200, 197

Thompson, R. R., Creech-Eakman, M. J., \& van Belle, G. T. 2002, ApJ, 577, 447

Weiner, J. 2004, ApJ, 611, L37

Weiner, J., Hale, D. D. S., \& Townes, C. H. 2003, ApJ, 588, 1064

Welch, D. L. 1994, AJ, 108, 1421

Whitelock, P., Marang, F., \& Feast, M. 2000, MNRAS, 319, 728

Willson, L. A. 2000, ARA\&A, 38, 573

Woitke, P., Helling, C., Winters, J. M., \& Jeong, K. S. 1999, A\&A, 348, L17

Wood, P. R., et al. 1999, in IAU Symp. 191, Asymptotic Giant Branch Stars, ed. T. Le Bertre, A. Lèbre, \& C. Waelkens (San Francisco: ASP), 151 